\title{
Holographic Microscopy with Python and HoloPy
}

\author{
Solomon Barkley, Thomas G. Dimiduk, Jerome Fung, David M. Kaz, Vinothan N. Manoharan, Ryan \\ McGorty, Rebecca W. Perry, and Anna Wang
}

\begin{abstract}
A holographic microscope captures interference patterns, or holograms, that encode three-dimensional (3D) information about the object being viewed. Computation is essential to extracting that 3D information. By wrapping low-level scattering codes and taking advantage of Python's data analysis ecosystem, HoloPy makes it easy for experimentalists to use modern, sophisticated inference methods to analyze holograms. The resulting data can be used to understand how small particles or microorganisms move and interact. The project illustrates how computational tools can enable experimental methods and new experiments.
\end{abstract}

Index Terms-G.1.8.g Inverse problems, I.4.0.b Image processing software, I.4.1.b Imaging geometry, I.5.1.e Statistical, J.2 Physical Sciences and Engineering

\section{INTRODUCTION}

$\mathrm{H}$ OLOGRAPHIC microscopy is an elegant way to image tiny objects in three dimensions. In its simplest form, a holographic microscope is the same as a conventional light microscope, except that the light source is a laser. The coherent light from the laser scatters from the sample and interferes with the transmitted light, producing a pattern of bright and dark fringes called a hologram, as shown in Figure 1 (although colloquially the term "hologram" often refers to a 3D image, we use it in its original sense of a 2D interference pattern). Traditionally, holograms were recorded on film, and the 3D information encoded in their fringes was reconstructed by shining light through the hologram. Gabor, the discoverer of holography, showed that reconstruction yields a 3D image because it reproduces the original field scattered from the sample, including both its amplitude and phase [6].

Nowadays holograms are recorded digitally, and the $3 \mathrm{D}$ information is reconstructed by simulating light shining through the image (Figure 2). Digital recording and reconstruction have enabled many scientific advances. For example, holographic time-series (or movies) recorded by high-speed digital cameras have been reconstructed to reveal how micro-organisms swim in three dimensions [10] and how tracer particles move in a turbulent flow [16]. Our research group does similar experiments. We record holograms of tiny colloidal particles or biological organisms moving in a fluid to understand how they organize themselves into more complex structures. To do that, we must determine their 3D positions in each hologram of a timeseries.

- Harvard John A. Paulson School of Engineering and Applied Sciences and Department of Physics, Harvard University, Cambridge, MA 02138. E-mail:vnm@seas.harvard.edu

- Thomas G. Dimiduk is currently at Tesla, Inc.

- Jerome Fung is currently at the Department of Physics, Wellesley College.

- David M. Kaz is currently at Agilent Technologies.

- Ryan McGorty is currently at the Department of Physics and Biophysics, University of San Diego.

- Rebecca W. Perry is currently at Wayfair, Inc.

- Anna Wang is currently at the Department of Molecular Biology, Massachusetts General Hospital.

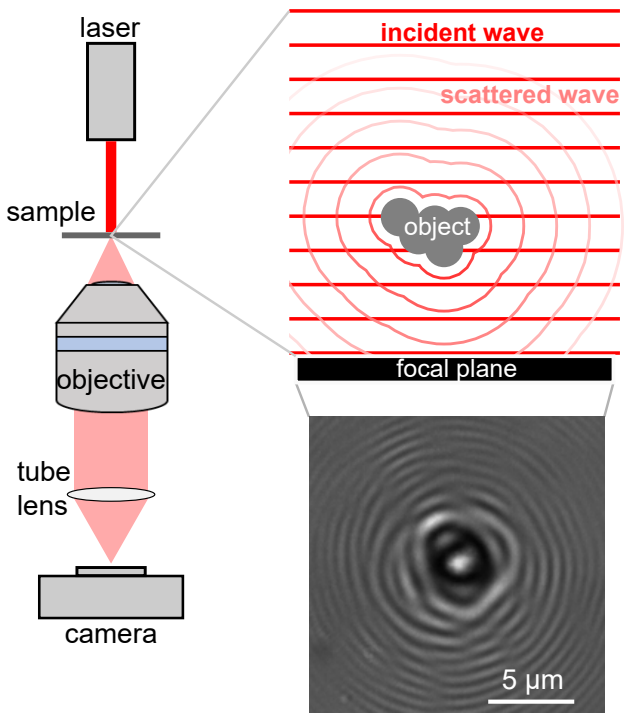

Fig. 1. An in-line holographic microscope (left) consists of a laser, an objective lens, and a camera. Some of the incident light is scattered by the sample and interferes with the transmitted light (upper right). The interference pattern recorded at the focal plane is called a hologram (lower right).

This task is complicated by two problems inherent to reconstruction. First, the reconstructed image can have unphysical artifacts [1], [7], [14], [15], such as the blurring and fringes shown in Figure 2. Second, the reconstruction must be further processed to extract information about the object, adding another layer of analysis.

A computational method has recently emerged that circumvents both of these problems. Starting with a model that can exactly simulate (to within numerical precision) the light scattered from microscopic objects, an algorithm adjusts the parameters in the model until the simulation matches, or fits, a measured hologram [9], [13]. Using this technique, we can infer the 3D positions of multiple objects directly from the hologram, without ever reconstructing the scattered field (Figure 2). Though computationally more 


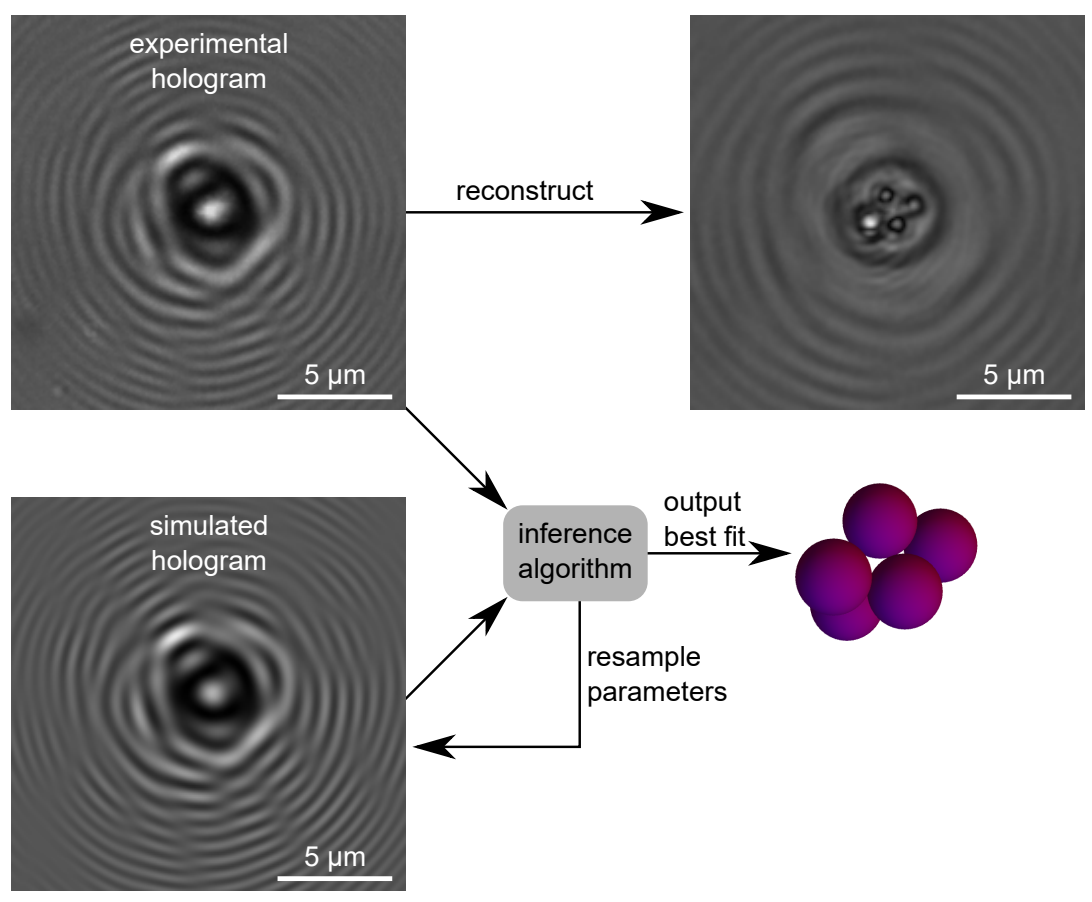

Fig. 2. Two computational methods to extract 3D information from an in-line hologram. Reconstruction (top) involves simulating light propagating through the hologram to yield a 3D image of the sample. From the reconstruction, parameters of interest, such as the positions of individual particles, can be measured by processing the image. This method tends to introduce artifacts such as blurring and fringe noise, which can be seen in the reconstructed image. Inference (bottom) involves iteratively simulating holograms and comparing them to data to extract information about the object imaged by the microscope.

demanding, this inference approach avoids the artifacts inherent to reconstruction while still taking advantage of the phase information encoded by the holograms.

The inference approach has transformed how our group does experiments. Previously, we needed to design the microscope so that the artifacts were minimized in 3D reconstructions. Now that we no longer rely on reconstruction, we can keep the instrument simple-no more than a conventional light microscope equipped with a laser and a digital camera-and use computers to handle the complex task of extracting the information we want.

Twelve years ago, we set out to make that complex task as simple as possible. We developed HoloPy, a Python package that simulates scattering and fits scattering models to measured holograms. Here we describe how Python made it possible to create an experimentalist-friendly package, and how we use it to analyze data from experiments in softmatter physics and biology.

\section{Why PYThON?}

We originally wrote HoloPy to numerically reconstruct holograms from our experiments. At the time (2006), many image processing codes were written in IDL or MATLAB. We chose Python because it was (and is) a general-purpose, cross-platform, free and open-source language; because we could write a numerical reconstruction routine in just a few lines of code using the NumPy package [12]; and because it was easy for us to learn. The last criterion was especially important for a burgeoning research group, since we needed to spend most of our time building instruments, making measurements, and thinking about physics-and not, in general, writing code.

Since we started using inference instead of reconstruction to analyze holograms, the package has changed a lot. But the focus on making it easy to use and modify has not. HoloPy is still written with experimental scientists in mind. Python has served us well in pursuit of this goal, because it allows us to wrap legacy codes in a common interface and to integrate existing Python packages to add functionality.

\subsection{Wrapping scattering codes}

When we moved to an inference framework, we needed HoloPy to simulate scattering from different kinds of objects. There were two possibilities: implement scattering models directly in Python or use existing implementations written in Fortran. It wasn't easy to write scattering models from scratch. The scattering from our samples is complicated because the size of the particles is comparable to the wavelength of light (about half a micrometer). Even the simplest scattering model-the Lorenz-Mie theory, which describes how a homogeneous sphere scatters light-requires calculating a host of special functions and evaluating series expansions with dozens of terms, all the while avoiding overflow or underflow errors [19]. We realized that there was no point in writing new code in Python when there were already well-tested Fortran codes that did all of these tasks efficiently. And there were codes for many different kinds of objects, including not only spheres but also collections of spheres, spheroids, cylinders, and even arbitrarily shaped particles.

However, each scattering code had its own coordinate system and interface, which were often cumbersome: pa- 
rameters were specified by input files in arcane formats or through command-line arguments. So we used Python to wrap the codes in an object-oriented interface with a common interface. The f2py system made it easy to wrap Fortran subroutines. For codes designed to run as executables rather than as subroutines, we wrote setup scripts to compile the executables and wrappers to communicate with them through Python's subprocess module, which works across all platforms. With this approach, new users needed to learn only one interface, which we could make intuitive and concise (see Figure 3).

\subsection{Integration with data analysis packages}

As we added more complex scattering models to HoloPy, we found that we needed to include more sophisticated methods to fit those models to the data. Fitting complex models involves varying many free parameters. A scattering model for a cluster of five colloidal particles, like that shown in Figure 2, might have 15 free parameters, corresponding to the positions of all the particles. Finding the parameters that best fit a hologram of this system involves a search in a 15-dimensional space.

To address this problem, we integrated a Python implementation for Markov-chain Monte Carlo (MCMC) calculations, emcee [4], with HoloPy. MCMC is a powerful method for fitting models with many parameters to data, and emcee is an implementation that can handle many different kinds of problems. Integrating emcee with HoloPy allowed us to define a concise interface and to provide sensible defaults specifically for analysis of holograms. It was easy to integrate the two packages, since both were written in Python.

Although packages like emcee didn't drive us to start working in Python, we now benefit from Python's growing prominence in the data science community. There are now many packages for MCMC and visualization that we can use to enhance HoloPy.

\section{A BRIEF TOUR OF HOLOPY}

Here we show some examples of calculations in HoloPy to illustrate how the scattering models and inference frameworks are integrated.

\subsection{Simulating a hologram from a given object}

A scattering model is essential to the inference approach. Figure 3 shows how to simulate a hologram using HoloPy's built-in models. We first tell HoloPy about the shape of our camera, the size of its pixels, and the properties of the incident illumination - its wavelength, polarization, and the refractive index of the medium it travels in. Then we define an object. In the example, we define a spherical particle using five parameters: its center position $(x, y, z)$, radius $r$, and refractive index $n$. Given this information, HoloPy calculates the scattered field at the camera. It then numerically calculates the interference between the scattered and transmitted waves to simulate the hologram.

Simulations like these are a convenient way to explore how holograms change for different particles or different parameters. For example, it's easy to change the particle from a sphere to an ellipsoid, as shown in Figure 3. We don't have to specify the scattering model when we do this. We define our object, and HoloPy automatically selects the appropriate model: the Lorenz-Mie theory for the sphere, and the T-Matrix formulation [11] for the ellipsoid.

\subsection{Inferring an object's properties from a hologram}

The real power of simulation lies in solving the inference (or inverse) problem: given a measured hologram, determine the position and properties of a microscopic particle. A sample inference calculation is shown in Figure 4. We specify the following: (1) variables describing the incident illumination; (2) an experimentally measured hologram (data_holo) and an estimate of the noise in that hologram; (3) the expected shape of the particle (here, a Sphere), along with parameters of the particle to vary, and over what ranges we expect them to vary; (4) an initial guess for the free parameters; (5) a complete model for the simulation, which includes a scattering theory (which we let HoloPy choose automatically) and a model for the optical train of the microscope (here called AlphaModel [9]); (6) an inference algorithm, along with settings for that algorithm (here, we use emcee and let HoloPy choose the default settings from TemperedStrategy ( ) ). With this information, HoloPy determines the best fit, as shown in Figure 4. It also outputs a results object that contains a full description of the model and the inference strategy as well as the fitted parameter values.

Although what happens behind the scenes is complex, the code in Figure 4 is not. In keeping with our goal of making complex tasks simple, HoloPy chooses intelligent defaults for the MCMC algorithm and the scattering model. Also, the object hierarchy makes the calculation modular. It is straightforward to change, for example, the inference strategy while keeping the scattering model fixed, or vice versa.

\section{EXPERIMENT MEETS COMPUTATION}

We've given only a taste of HoloPy above. Additional functionality to manage and analyze experimentally acquired holograms is central to the package. Here we explain how we designed this functionality to meet the needs of experimentalists.

\subsection{Enabling sophisticated data analysis}

In a typical experiment we quantify not only the positions of particles, but also the uncertainty on those positions. The uncertainty is primarily related to the noise in the hologram. We care about the uncertainty because we ultimately want to understand how a particle moves or interacts, and the uncertainty tells us how much to trust our measurements.

HoloPy uses a Bayesian framework to determine the uncertainty. Given the scattering model, measured hologram, and estimate of the noise in that hologram, it constructs a posterior probability distribution for the free parameters in the model [2]. Because the posterior is a function of many parameters, it's inefficient to calculate it on a regular grid spanning the whole parameter space. Instead, the MCMC framework randomly samples sets of parameters from the 


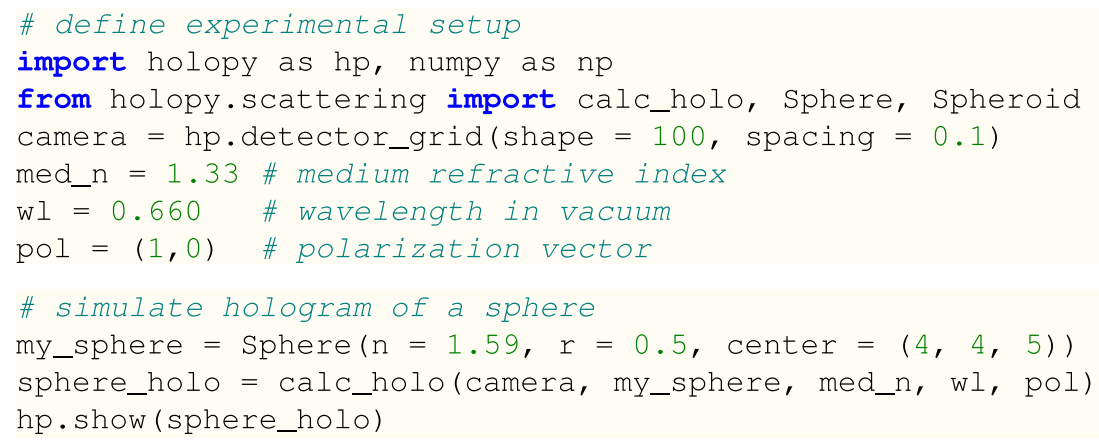

Fig. 3. HoloPy code to simulate holograms produced by a $1-\mu \mathrm{m}$ polystyrene sphere and a $2 \times 1-\mu \mathrm{m}$ ellipsoid, both in water. Simulated holograms are shown at right. Note that HoloPy is agnostic to the units of length, as long as they are consistent. Here, all lengths are in $\mu \mathrm{m}$.

posterior. For each set of parameters (or "sample"), HoloPy simulates the hologram and calculates the difference between the simulated and measured holograms. The MCMC algorithm uses this difference to choose the next sample. The distribution of all the samples tells us the best-fit parameters as well as their uncertainties and covariances (Figure 5).

The Bayesian framework is particularly well-suited to experiments on microscopic particles, for which properties such as the size or refractive index are usually known only approximately. The most convenient way to specify this "fuzzy" knowledge is through a probability distribution, or prior. In the example in Figure 4, we use a Gaussian prior for the refractive index of the sphere and a bounded Gaussian prior for the radius.

Because the MCMC analysis returns all the samples, and not just the best-fit parameters, we can visualize and characterize the posterior probability density. In Figure 5, we plot the samples for the refractive index $(n)$ and radius $(r)$ from an MCMC analysis of a hologram of a single sphere. Each point represents a particular set of parameters-or, equivalently, a simulated hologram. From these samples, we can construct distributions using histograms or kernel density estimates with the seaborn package (https:// seaborn.pydata.org). The plots allow us to characterize the uncertainty. We see, for example, that our estimates of $n$ are correlated with our estimates of $r$, which tells us that a measured hologram is equally well fit by a small radius and large index as by a large radius and small index. That makes sense physically: the effective size of the particle that the light "sees" is related to the product of $n$ and $r$. We can also construct marginal distributions for single parameters such as $x, y$, or $z$. These plots give us the uncertainty in each parameter, after marginalizing (accounting for) the uncertainties and correlations among all the other parameters. With this wealth of information about the uncertainties, we can optimize the experiment or propagate the uncertainties to test physical models of how the particles move.

MCMC methods are powerful tools for Bayesian inference, but they come at a cost. A typical MCMC calculation might involve thousands of samples, which means HoloPy has to simulate thousands of holograms for each measured one. The number of samples is the price we must pay for accurate uncertainty estimates.

That price can be made smaller through a few computational tricks that we built into HoloPy. Simulating a $512 \times 512$-pixel hologram involves making 262,144 individual scattering calculations, which takes (in total) about a second on a modern CPU. Since the MCMC algorithm does thousands of simulations for each fit, we realized that we could save a significant amount of computational time by reducing the number of pixels in each MCMC step. The simplest way to do this is to choose a random set of pixels in the measured hologram and fit just those pixels, rather than the whole hologram [3]. We found that we could get accurate results with only tens of pixels, which speeds up the calculation by a factor of 100 or more. This strategy works because the hologram contains a lot of redundant information in its fringes. We tell HoloPy to use random subsetting through the Strategy object.

These are just a few ways in which HoloPy enables the kinds of data analysis that experimentalists need. While some of the functionality, such as random subsetting, is built into HoloPy, the rest comes from the broader Python ecosystem, including packages like emcee and seaborn.

\subsection{Time-series analysis}

Python's extended infrastructure also comes in handy for time-series analysis. We record time-series because we are interested not only in the size and shape of microscopic particles, but also in understanding how they move. Tracking the motion of a bacterium tells us about how it searches for 

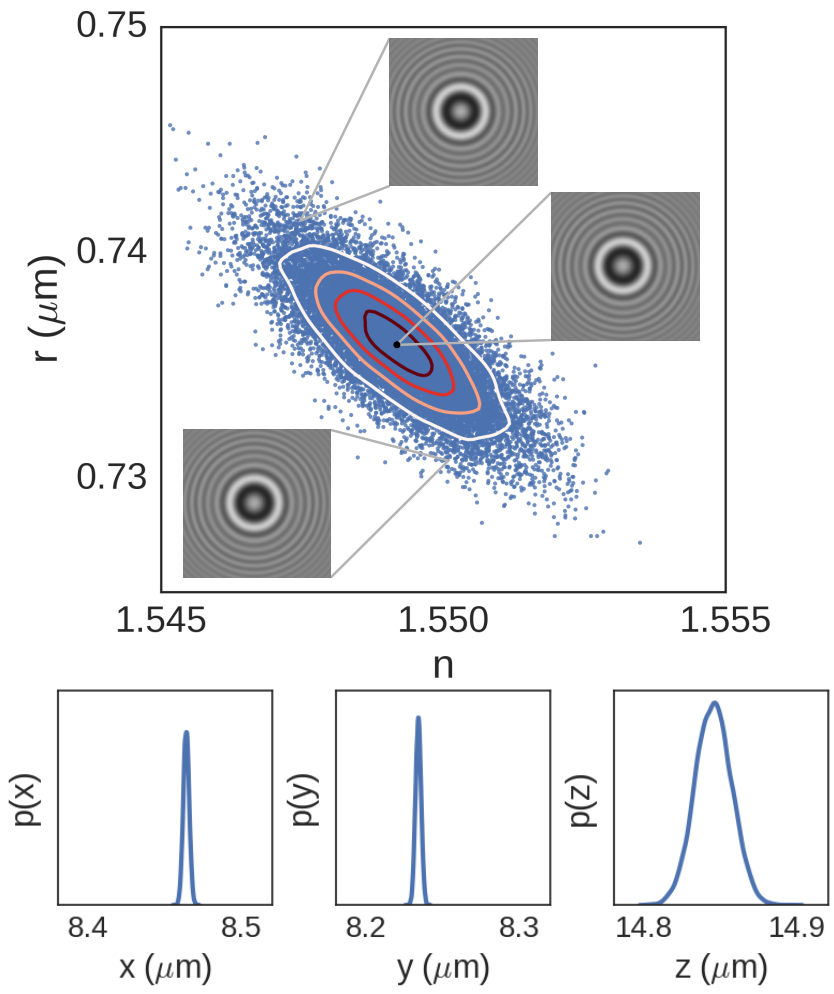

Fig. 5. An MCMC calculation returns many sets of parameter values, each corresponding to a simulated hologram. Here we show visualizations of the MCMC samples returned from analysis of the hologram shown in Figure 4. The joint distribution at top provides a measure of the correlations between fitted parameters, while the fully marginalized distributions at bottom characterize the uncertainties of each parameter in isolation (bottom). The top diagram also shows the contour lines of a kernel density estimate of the joint distribution, as well as the simulated holograms corresponding to the best fit (center) and two other samples.

in a format that supports arbitrary metadata. We use either HDF5 or TIFF containers.

We also had to figure out how to include metadata in HoloPy's internal data structures. NumPy arrays cannot handle metadata. We tried to use Pandas DataFrame, a versatile container for heterogeneous data, but we found it difficult to adapt to time-series of holograms. We ultimately decided to use the xarray package [8], which was designed to handle such multidimensional data sets with metadata.

Internally, HoloPy stores holograms and metadata in xarray.DataArray objects. This format has two advantages over a traditional NumPy array. First, we can assign coordinates to each point in an image and access parts of the image according to their coordinates instead of pixel indices. This feature is particularly useful because it matches how we think about holograms-in terms of regions of space rather than pixel numbers. Second, we can associate metadata, such as illumination wavelength and polarization, in the xarray.DataArray.attrs property, which is a Python dictionary.

During calculations, HoloPy extracts numerical data from the xarray.DataArray objects and treats it as a simple array, then repackages the results into an xarray.DataArray before returning it. With this approach, calculations proceed at the speed of NumPy while still preserving the metadata. Metadata is also preserved in image processing tasks such as cropping, background division, or normalization.

\section{BEHIND THE SCENES}

Because experimentalists don't have a lot of time to write code, we made it easy to adapt HoloPy to new experimental systems or new analysis techniques. The object hierarchy (Figure 4) makes it straightforward to add new components such as scattering theories, scattering models, and inference strategies.

\subsection{Adding new scattering theories}

The core functionality of HoloPy is a universal interface to scattering models. HoloPy currently contains distinct models for single spheres, collections of spheres, axisymmetric objects, and arbitrary voxelated objects. The input to each of these models is the same. Given descriptions of the object and the camera or detector, HoloPy selects the scattering model most appropriate for the object and asks the model to calculate the scattering at the coordinates specified by the detector. It does this by transforming those coordinates into whatever coordinate system the model uses. It then adds the scattered field coherently to the transmitted field to simulate the hologram. Because the scattered fields from each model are converted to a Python object, HoloPy can use the same code (a few lines of NumPy array manipulations) to calculate the hologram from the scattered-field object, irrespective of the model.

With this modular architecture, we need only define a new class derived from the ScatteringTheory base class to add a new scattering model to HoloPy. The new class must define a method that takes inputs of a HoloPy Scatterer object and an array of coordinates and returns scattered fields at the coordinate locations. This minimal requirement gives us the flexibility to wrap scattering codes in multiple ways. Scattering theories for single and multiple spheres are compiled into HoloPy modules with the help of f2py. The scattering theory for axisymmetric objects is compiled into an executable file that HoloPy runs after writing arguments to a temporary file. And HoloPy interacts with a command-line interface implementation of the discrete dipole approximation [20] through Python's subprocess module. Because the overhead of spawning subprocesses is small compared to the time required to do scattering calculations, this approach is convenient and not too costly, except when calculating small holograms or small subset fractions.

\subsection{Validation}

Since the interface to the scattering codes is a core part of HoloPy, we must test that it returns correct results. We did not write many of these codes ourselves, and we must ensure that they compile properly and return the expected results on different platforms. HoloPy therefore includes a unit testing framework that tests whether the scattering models output the correct results for a variety of different conditions.

We've found these tests to be extremely useful when modifying HoloPy, and particularly when adding a new 
scattering model. Many such models can handle spheres as a test case. So, when we added scattering models for ellipsoids and for clusters of spheres, we were able to validate the results of these models against our trusted Lorenz-Mie model.

\section{CONCLUSION}

In our 12 years of working on HoloPy, we've learned a lot about bridging experiment and computation. For example, we've learned that modularity encourages new science. Although it's not always easy to wrap a new scattering code, HoloPy's modularity makes it easy to use once it has been wrapped. As a result, we can spend more time thinking about what physical phenomena we want to look at and less about how we'll analyze the data. For example, after we included a scattering model for non-spherical colloidal particles, we realized that we could use the same model for rod-like bacteria. That realization led to an experiment that revealed some new aspects of bacterial motion [18]. The experiment wouldn't have happened had it not been easy to use the same model to analyze two completely different systems.

We've also found that it's more important for calculations to be expeditious than efficient. We generally optimize our models only when it takes more than a few days to analyze the data from an experiment, because that's the typical timescale of a new experiment. While we're waiting for the analysis from one experiment, we can do another. If necessary, we can get results from the analysis more quickly by using approximate methods like random subsetting. For us, it's more important that calculations can be set up and started quickly than finished quickly. A day spent writing code to do the analysis is a day that could have been spent doing more experiments. But a day that the computer spends analyzing data doesn't cost us any time in the lab.

That's not to say efficiency isn't important. In fact, figuring out how to parallelize inference calculations for timeseries is one of our goals for future versions of HoloPy. Although scattering calculations for a single hologram can be parallelized by pixel using graphics processing units (GPUs) or multiple processor cores, parallelizing by frame is more challenging. For a time-series, the best way to get a good guess for one frame is to use the results of the previous one. As a result, time-series are more naturally analyzed sequentially than in parallel. We also have plans to analyze holograms from larger and more complex objects like living cells. Simulating scattering from these systems requires complex models with lots of parameters. In both cases, new methods to find good initial guesses for the parameters could dramatically speed up the calculations. One potential approach involves new optimization tools such as the Python package Simple (https://github.com/ chrisstroemel/Simple). Such techniques can generate an initial guess quickly by estimating the maximum of the posterior probability density from a few points in parameter space.

We welcome bug reports, feedback, and contributions to HoloPy. The source code is licensed under the GNU General Public License version 3 and is hosted on GitHub (https://github.com/manoharan-lab/holopy). For convenience, we also distribute binary packages of HoloPy (with all the scattering codes already compiled) through the conda-forge channel for the Anaconda Python distribution. The conda package is cross-platform and is automatically updated whenever a new version is released. New users can get started with HoloPy by running conda install $-c$ conda-forge holopy and following the examples in the tutorial at http://holopy.readthedocs.io.

\section{ACKNOWLEDGMENTS}

We acknowledge support from the National Science Foundation through grant numbers DMR-1306410 and DMR1420570 and from an Alfred P. Sloan Research Fellowship.

\section{REFERENCES}

[1] Fook Chiong Cheong, Bhaskar Jyoti Krishnatreya, and David G. Grier. Strategies for three-dimensional particle tracking with holographic video microscopy. Optics Express, 18(13):13563-13573, 2010.

[2] Thomas G. Dimiduk and Vinothan N. Manoharan. Bayesian approach to analyzing holograms of colloidal particles. Optics Express, 24(21):24045-24060, 2016.

[3] Thomas G. Dimiduk, Rebecca W. Perry, Jerome Fung, and Vinothan N. Manoharan. Random-subset fitting of digital holograms for fast three-dimensional particle tracking. Applied Optics, 53(27):G177-G183, 2014.

[4] Daniel Foreman-Mackey, David W Hogg, Dustin Lang, and Jonathan Goodman. emcee: the MCMC hammer. Publications of the Astronomical Society of the Pacific, 125(925):306, 2013.

[5] Jerome Fung, K. Eric Martin, Rebecca W. Perry, David M. Kaz, Ryan McGorty, and Vinothan N. Manoharan. Measuring translational, rotational, and vibrational dynamics in colloids with digital holographic microscopy. Optics Express, 19(9):8051-8065, 2011.

[6] Dennis Gabor. A new microscopic principle. Nature, 161(4098):777-778, 1948.

[7] Dennis Gabor. Microscopy by reconstructed wave-fronts. Proceedings of the Royal Society of London A: Mathematical, Physical and Engineering Sciences, 197(1051):454-487, 1949.

[8] Stephan Hoyer and Joseph Hamman. xarray: N-D labeled arrays and datasets in Python. Journal of Open Research Software, 5(1), 2017.

[9] Sang-Hyuk Lee, Yohai Roichman, Gi-Ra Yi, Shin-Hyun Kim, Seung-Man Yang, Alfons van Blaaderen, Peter van Oostrum, and David G. Grier. Characterizing and tracking single colloidal particles with video holographic microscopy. Optics Express, 15(26):18275-18282, 2007.

[10] Nancy I. Lewis, Wenbo Xu, Stefan K. Jericho, Hans J. Kreuzer, Manfred H. Jericho, and Allan D. Cembella. Swimming speed of three species of alexandrium (dinophyceae) as determined by digital in-line holography. Phycologia, 45(1):61-70, 2006.

[11] Michael I. Mishchenko and Larry D. Travis. Electromagnetic Scattering by Nonspherical Particles. In Rodolfo Guzzi, editor, Exploring the Atmosphere by Remote Sensing Techniques, number 607 in Lecture Notes in Physics, pages 77-127. Springer Berlin Heidelberg, 2003.

[12] Travis E. Oliphant. A guide to NumPy. Trelgol Publishing, USA, 2006.

[13] Ben Ovryn and Steven H. Izen. Imaging of transparent spheres through a planar interface using a high-numerical-aperture optical microscope. Journal of the Optical Society of America A, 17(7):12021213, 2000.

[14] Ye Pu and Hui Meng. Intrinsic aberrations due to mie scattering in particle holography. JOSA A, 20(10):1920-1932, 2003.

[15] Ye Pu and Hui Meng. Intrinsic speckle noise in off-axis particle holography. JOSA A, 21(7):1221-1230, 2004.

[16] J. Sheng, E. Malkiel, and J. Katz. Using digital holographic microscopy for simultaneous measurements of 3D near wall velocity and wall shear stress in a turbulent boundary layer. Experiments in fluids, 45(6):1023-1035, 2008. 
[17] The HDF Group. Hierarchical Data Format, version 5, 1997-2018. http:/ /www.hdfgroup.org/HDF5/.

[18] Anna Wang, Rees F. Garmann, and Vinothan N. Manoharan. Tracking E. coli runs and tumbles with scattering solutions and digital holographic microscopy. Optics Express, 24(21):2371923725, 2016.

[19] W. J. Wiscombe. Improved Mie scattering algorithms. Applied Optics, 19(9):1505, 1980.

[20] Maxim A. Yurkin and Alfons G. Hoekstra. The discretedipole-approximation code ADDA: capabilities and known limitations. Journal of Quantitative Spectroscopy and Radiative Transfer, 112(13):2234-2247, 2011. 